\title{
Pre-eclampsia: a Life-threatening Pregnancy Syndrom
}

\author{
Andrea Brazdova ${ }^{1,2 *}$, Alena Keprova ${ }^{3}$, Jarmila Zidkova ${ }^{2}$ and Jindrich Madar $^{4}$ \\ ${ }^{1}$ Cordelier Research Center; Pierre and Marie Curie University; Paris - France. ${ }^{2}$ Department of Biochemistry and \\ Microbiology; Institute of Chemical Technology Prague; Prague - Czech Republic. ${ }^{3}$ Institute of Organic Chemistry \\ and Biochemistry; Academy of Sciences of the Czech Republic; Prague - Czech Republic. ${ }^{4}$ Institute for the Care of \\ Mother and Child; Prague - Czech Republic
}

\begin{abstract}
Pre-eclampsia is a serious pathological state affecting 5-10\% of pregnant women. Currently, it is diagnosed in the second half of pregnancy, particularly after the 20th week. Symptoms mostly correspond to the changes of blood vessels and kidneys. The severity of pre-eclampsia is proportional to symptomatic manifestations, thus the more symptoms present, the higher is of pre-eclampsia development. Although there are several studies dealing with preeclampsia pathology, the complete etiology is still unknown. In this review paper, several theories are presented and discussed.
\end{abstract}

Key words: placenta, pre-eclampsia, pregnancy, oxidative stress

\section{INTRODUCTION}

Pre-eclampsia (PE) is a disorder affecting both the mother and the unborn child. It occurs only during the pregnancy and may persist until the postpartum period. Depending on the nature of the disease, it may be distinguished into the mild and severe form. In some cases, early and late type of PE is indicated and they are discussed as separate entities. Mild PE is defined by hypertension $(\geq 140 / 90 \mathrm{mmHg}$ ) on two occasions (at least $6 \mathrm{~h}$ apart) without the evidence of end-organ damage, and the presence of $300 \mathrm{mg}$ of proteins in a $24 \mathrm{~h}$ collected urine sample but no other symptoms affecting either mother or baby. Severe PE is characterized by blood pressure over 160/110 $\mathrm{mmHg}$ and may lead to liver/renal failure, disseminated intravascular coagulopathy and central nervous system abnormalities. If PEassociated seizures are experienced, the disorder develops into eclampsia. Premature births, induced by PE, are the major cause of maternal, neonatal morbidity and the increased mortality of newborns. The incidence rate varies from 5 to $8 \%$ of all the pregnancies in developed European countries (Arbogast and Taylor 1996; Preeclampsia foundation 2013).

Eclampsia and HELLP syndrome (hemolysis, elevated liver enzymes, low platelet count) may be an outcome of PE. This complex pathological condition is most often characterized by the rise in blood pressure, which can result in seizures, cerebral stroke, multiple organ failure and death of the mother and/or child. Swelling, sudden weight gain, headache and visual disturbance are less frequent symptoms. PE occurs after the 20th week of pregnancy and is therefore not diagnosed until the second half of pregnancy based on emerging clinical symptoms. In rare cases, it may occur before the 20th week. Although the etiology is unclear, several PE risk factors are known. The most common manifestations include

*Author for correspondence: andreabrazdova@ centrum.cz 
hypertension, edema and proteinuria. However, the higher permeability of placenta is also associated with PE. The higher permeability of reactive oxygen species (ROS) through the placenta may lead to the development of oxidative stress (OS) (Arnostova et al. 2007; Escudero and Sobrevia 2008; Pre-eclampsia foundation 2013).

Several theories have been suggested to explain the origin of PE, including the hypothesis of uteroplacental ischemia (changes in the biology of placenta), immune disorders (systemic inflammatory response), genetic predispositions, malfunction structures of protein/lipoprotein disorders, and the hypothesis of oxygen radicals (Anthony and Johanson 1996). The hypothesis of uteroplacental ischemia is based on the expression of cytotrophoblastic adhesive molecules. It is suggested that the reduced penetration of substances through placenta leads to fetal damage and the release of toxic substances with some regulatory factors. A common pathological finding is a placenta with multiple surface infarcts (Mechurova 2002).

The current theory concerning the pathophysiology of PE relates a lack of trophoblast invasion into the wall of the spiral arteries. It is linked to the hypothesis of systemic inflammatory response supported by the fact that the fetoplacental unit contains paternal antigens that are capable of slowing down the trophoblast invasion. The hepatic expression of human leucocyte antigen G (HLA-G) may inhibit trophoblast invasion in uterine smooth muscle. Subsequently, the activated leukocytes release an elastase and attack an endothelium (Mechurova 2002; Stara 2012). Genetic hypothesis is based on the genetic predisposition of daughters whose mothers have a verifiable PE history. In this case, the risk of disease increases up to $22 \%$, for sisters up to 39\% (Mechurova 2002). The PE patients have a higher concentration of triglyceride and the lipoproteins of very low density (VLDL) in the plasma. VLDL hypothesis derives from the endothelial lining of the vascular damage effect of VLDL. The most discussed theory is that of OS. The balance between the oxygen radicals and antioxidants is disrupted during PE development. Natural antioxidant ability is reduced, which may lead to placental OS. This hypothesis is supported by the fact that the placenta in PE patients exhibits discontinuous perfusion due to the reduced pathological trophoblast invasion into the maternal vascular system. The above mentioned $\mathrm{OS}$ is considered as a significant consequence causing the reduced placental permeability (Borzychowski et al. 2006).

\section{PATHOLOGY OF PRE-ECLAMPSIA}

Nowadays, the PE syndrome is considered as a two-stage disease. The first stage is defined by the reduced placental permeability (specific proteins, placental 'debris') associated with abnormal implantation and fetal placental release into the maternal circulation. The malfunction of placenta may, in some cases, induce hypertension, renal impairment, proteinuria, damage of other organs and HELLP syndrome. The second stage is the maternal reaction to this condition. It is characterized by inflammation and endothelial dysfunction of parental cells (Redman and Sargent 2000). One of the fundamental pathophysiological changes is the so-called firm connection of fetal and maternal placenta. Pathological examination of placentas has shown placental infarction, narrowing of arteries and blood vessels, fibrin deposition and venous thrombosis. Placental ischemia induces hypertension, proteinuria, which is also associated with the deposition of fibrous material in the glomerular tubules, called glomerular endoteliosis (Baumwell and Karumanchi 2007).

Recent studies deal with the proteins that are produced by placenta and inhibit angiogenesis. Vascular endothelial growth factor (VEGF) and placental growth factor (PIGF) are pro-angiogenic factors that are involved in trophoblast proliferation (Grill et al. 2009). The growth factors and their receptors originate in the placenta. Placental cells also secrete the soluble isoform receptor (sFlt-1), which is generated through an alternative mRNA splicing. Soluble Flt-1 isoform has a role as an anti-angiogenic factor. It neutralizes the effect of PIGF and VEFG. The over-expression of sFlt-1 is associated with placental ischemia and hypoxia (Clark et al. 1998). Cooper et al. (1996) reported that VEGF and PIGF concentration were lower in the second trimester in women with late $\mathrm{PE}$ in comparison with the physiological pregnancies. This fact is most significant in the patients with severe PE, where VEGF and PIGF amount is reduced in comparison with the women with mild PE (St-Jacques et al. 1994). 
Maternal immune disorders play an important role in the pathophysiology of PE. Trophoblasts contain a wide range of immunogenic agents, such as HLA-G. This glycoprotein is responsible for the low trophoblast immunogenicity along with the high resistance to the maternal immune reaction. The presence of trophoblast with an insufficient HLA-G expression can activate the incompatible decidual immune cells in the uterine cavity (Matthiesen et al. 2005). The placental vascular wall is an integrated organ, composed of endothelial cells, smooth muscles and fibroblasts. Endothelial cells contain the surface receptors for thrombin, clotting factors, angiotensin II, and many more. Cytokines (e.g. interferon-gamma) and various types of ROS mediate an endothelial dysfunction. Clinical symptoms usually appear after the 20th week of pregnancy. Endothelial dysfunction may result in the increased permeability of endothelial cells, activation of platelets and the coagulation cascade, shifting the balance of vasoactive mediators, and thus favoring vasoconstriction and the development of OS (Davidge 1998).

\section{CLINICAL MANIFESTATION OF PRE- ECLAMPSIA}

The clinical manifestation varies since $\mathrm{PE}$ is associated with several risk factors (obesity, molar pregnancy, hypercoagulation, formation of clots, diabetes mellitus). The prevalence of PE increases up to $66 \%$ if there is already an existing chronic kidney disease (Baumwell and Karumanchi 2007). It was found that male seminal plasma antigens, expressed on trophoblasts, reduce the risk of PE. The major clinical manifestation includes hypertension, proteinuria, edema, hyperuricemia and HELLP syndrome. Blood pressure is a function of peripheral vascular resistance and cardiac output. According to the World Health Organization (WHO), hypertension is considered as a blood pressure of $140 / 90 \mathrm{mmHg}$ or higher at two separate measurements within $6 \mathrm{~h}$ (Hajek 1999). Proteinuria means the increased amount of total proteins in the urine. Glomerular membrane damage and reduced protein absorption in the back tubules cause an increase of up to $300 \mathrm{mg}$ in $24 \mathrm{~h}$ (Brown et al. 2001). Swelling is caused by the increased protein loss or the inadequate intake of fats and sugars. In the advanced stage of pregnancy, it may be caused by obstruction due to enlarged veins in the uterus (Felfernig-Boehm et al. 2000).

Hyperuricemia is defined as the increased level of uric acid in the blood. If the level is higher than $320 \mu \mathrm{M} / \mathrm{L}$, it becomes one of the primary PE indicators. The most significant cause of hyperuricemia is the insufficient feedback resorption of uric acid in renal tubules (Cai et al. 2009). HELLP syndrome differs from PE by hemolysis, the increased activity of liver enzymes and decreased level of platelets. HELLP is diagnosed in $20 \%$ of severe PE with the so-called thrombotic microangiopathy, which is defined as a syndrome involving the occurrence of clots in the veins, acute hemolytic anemia and thrombocytopenia (Felfernig-Boehm et al. 2000; Stara 2012).

\section{CLINICAL SCREENING FOR PRE- ECLAMPSIA}

The PE markers include increased blood pressure, elevated or decreased protein in the urine, insufficient cerebral perfusion (headache, dizziness, tremor), nausea, vomiting, epigastric pain, distorted/double vision. Nowadays, the laboratory screening includes the monitoring of malondialdehyde levels, uric acid, fibronectin, human chorionic gonadotropin (hCG), increase in hemoglobin and hematocrit and the enzyme activity of selected enzymes (aspartate aminotransferase, alanine aminotransferase and lactate dehydrogenase). Malondialdehyde, which is a lipid peroxidation product, can be used as a new PE marker (Hajek 1999). Purine level is dependent on the influence of oxygen radicals. Although the released fibronectin from damaged vascular endothelial cells could theoretically contribute to PE prediction, this marker may easily provide false-positive results (Friedman and Schiff 1995).

\section{ASSOCIATION OF OXIDATIVE STRESS AND PRE-ECLAMPSIA}

OS is an imbalance between the free radicals and ROS and their elimination by antioxidants. OS represents a disproportion between the oxidants and the antioxidants. This misbalance leads to cell 
damage that may influence the entire body (Durackova 2010). ROS are described as the molecules of independent existence. Free radicals, with their unpaired electron, exhibit a high chemical reactivity towards other organic molecules. The main source of free radicals is the mitochondrial respiratory chain. It is known that 1$4 \%$ of oxygen is converted to superoxide and hydrogen peroxide during oxidative phosphorylation. Another source of free radicals is biotransformation process in the endoplasmic reticulum. The exogenous factors, which significantly increase the production of free radicals, are UV radiation, smoking, industrial pollution, pesticides, some pharmaceuticals and many organic solvents. ROS can cause intracellular reactions with lipids, DNA and proteins. These interactions can lead to the loss of cell permeability/genome stability and also other cellular functions (Cadenas and Sies 1998). PE causes hypoperfusion, placental ischemia and hypoxia and also reduces placental permeability so that reactive oxygen compounds are intensively produced. A reduced placenta can release various mediators such as the hCG hormone, cytokines, reactive oxygen and nitrogen species (nitrous oxide, hydrogen peroxide, superoxide anion). These mediators are detected when endothelial dysfunction occurs (Llurba et al. 2004). The throughput of reactive oxygen increases along PE developing during the pregnancy. This may lead to OS (Dennery 2010). The most important enzymatic oxidants are catalase, superoxide dismutase, glutathione-S-transferase, glutathione reductase and glutathione peroxidase (Kaur et al. 2008).

\section{CONCLUSIONS}

Albeit the vast advances in research and knowledge of PE during recent times, the basic research is still lacking behind. This lifethreatening syndrome is the fatal culprit of perinatal morbidity/mortality, abortions or premature births. It is suggested that an inadequate trophoblast invasion leads to a reduced placental perfusion at the end of the first trimester, which is followed by endothelial dysfunction and clinical manifestation by the end of the second/during the third trimester. Despite the therapeutical progress, the diagnosis is still very complicated. Currently, the diagnosis of PE is based on clinical symptoms that are noticeable at the end of the second trimester. Therefore, great attention should be paid on the biochemical actions associated with the development of PE. The monitoring of biochemical markers could contribute to a better understanding and be helpful for early diagnosis. While the main causes of PE are still unknown, the current studies on biochemical processes associated with PE require much more attention and financial support.

\section{ACKNOWLEDGEMENT}

This work was supported by the Grant Agency of the Czech Academy of Sciences (IAA 600110902) and by a specific university research (MSMT No 20/2013). The authors are grateful to MSc. Thomas Michl (Ian Wark Research Institute University of South Australia) and Mr. Brendan Fallon (Pierre and Marie Curie University, Paris, France) for proofreading the article.

\section{REFERENCES}

Antony J, Johanson RB. Critical care in pregnancy. Curr Obstet Gynae. 1996; 6: 98-104.

Arbogast BW, Taylor RN. Molecular mechanism of pre-eclampsia. 1st ed. Texas, USA: Medical Intelligence Unit. R.G. Landes company; 1996.

Arnostova A, Fialova L, Malbohan I, Zima T, Springer D. Pre-eclampsia and its diagnosis. Klin Bioch Metab. 2007; 15: 200-206.

Baumwell S, Karumanchi SA. Pre-eclampsia: Clinical manifestations and molecular mechanism. Nephron Clin Practice. 2007; 106: 72-81.

Borzychowski A, Sargent I, Redman C. Inflammation and pre-eclampsia. Semin Fetal Neonatal Med. 2006; 11: 309-316.

Brown MA, Lindheimer MD, Swiet M, Van Assche A, Moutquin JM. The classification and diagnosis of the hypertensive disorders of pregnancy: Statement from the international society for the study of hypertension in pregnancy. Hypertens Pregnancy. 2001; 20: 9-15.

Cadenas E, Sies H. The lag phase. Free Radic Res. 1998; 28: 601-609.

Cai Z, Xu X, Wu X, Zhou C, Li D. Hyperuricemia and the metabolic syndrome in Hangzhou. Asia Pac $J$ Clin Nutr. 2009; 18: 81-87.

Clark DE, Smith SK, He Y. A vascular endothelial growth factor antagonist is produced by the human placenta and released into the maternal circulation. Biol Reprod. 1998; 59: 154-158. 
Cooper JC, Sharkey AM, Charnock-Jones DS. VEGF mRNA levels in placenta from pregnancies complicated by pre-eclampsia. Brit J Obstet Gynecol. 1996; 103: 1191-1196.

Davidge ST. Oxidative stress and altered endothelial cell function in pre-eclampsia. Semin Reprod Endocrinol. 1998; 16: 65-73.

Dennery PA. Oxidative stress in development: nature or nurture? Free Radic Biol Med. 2010; 49: 1147-1151.

Durackova Z. Some current insights into oxidative stress. Physiol Res. 2010; 59: 459-469.

Escudero C, Sobrevia L. A hypothesis for preeclampsia: adenosine and inducible nitric oxide syntheses in human placenta microvascular endothelium. Placenta. 2008; 29: 469-483.

Felfernig-Boehm D, Salat A, Vogl SE, Murabito M, Felfernig M, Schmidt D, et al. Early detection of preeclampsia by determination of platelet aggregability. Tromb Res. 2000; 98: 139-146.

Friedman SA, Schiff E. Biochemical collaboration of endothelial involvement in severe pre-eclampsia. Am J Obstet Gynecol. 1995; 172: 202-203.

Grill S, Rusterholz C, Zanetti-Dallenbach R. Potential markers of pre-eclampsia - a review. Reprod Biol Endocrinol. 2009; 14: 7-70.

Hajek Z. Prenatal care in physiological pregnancies and serious pathologies. Mod Gynekol Porod. 1999; 8: 63-67.

Kaur G, Mishra S, Sehgal A, Prasad R. Alterations in lipid peroxidation and antioxidant status in pregnancy with pre-eclampsia. Mol Cell Biochem. 2008; 313: $37-44$.
Llurba E, Gratacos E, Martin-Gallan P, Cabero L, Dominguez C. A comprehensive study of oxidative stress and antioxidant status in pre-eclampsia and normal pregnancy. Free Rad Biol Med. 2004; 37: 557-570.

Matthiesen L, Berg G, Ernerudh J, Ekerfelt Ch, Jonsson Y, Sharma S. Immunology of pre-eclampsia. Chem Immunol Allergy. 2005; 89: 49-56.

Mechurova A. The interval between pregnancies and the risk of pre-eclampsia. New Eng J Med. 2002; 346: $1-33$.

Pre-eclampsia Foundation Online [homepage on the internet]. Melbourne: Pre-eclampsia Foundation; 2013 [cited 2013 Aug. 24]. Available from: http://www.pre-eclampsia.org.

Redman CWG, Sargent IL. Placenta debris, oxidative stress and pre-eclampsia. Placenta. 2000; 21: 591602.

Stara T. The study of selected pathophysiological factors of human pre-eclampsia [diploma thesis]. Prague, Czech Republic: Institute of Chemical Technology; 2012.

St-Jacques S, Forte M, Lye SJ, Letartem M. Localization of endoglin, a transforming growth factor beta binding protein, and of CD44 and integrins in placenta during the first trimester of pregnancy. Biol Reprod. 1994; 51: 255-259.

Received: October 01, 2013; Accepted: April 24, 2014. 